\title{
RESÚMENES DEL XIV CONGRESO PERUANO \\ DE ENDOCRINOLOGÍA, 2014 \\ PRESENTACIÓN
}

La Sociedad Peruana de Endocrinología, fundada en 1957 y reconocida como Sociedad Científica por el Colegio Médico del Perú (Registro: 571127-SM-24), tiene entre sus principales finalidades la de promover y desarrollar la investigación científica médica en el ámbito de la endocrinología y sus subespecialidades, garantizando que no se transgredan las consideraciones éticas en los protocolos de investigación, y promover y desarrollar el intercambio médico científico a nivel local, nacional e internacional, así como la información y educación en salud en el ámbito endocrinológico.

De acuerdo a este marco directriz, organiza cada año un gran evento científico nacional, el último de los cuales correspondió al XIV Congreso Peruano de Endocrinologia, realizado en la ciudad de Lima del 7 al 9 de agosto de 2014, en las instalaciones del Centro de Convenciones del Colegio Médico, el cual contó con la participación de 12 expositores extranjeros y 20 nacionales, durante el cual se revisó los adelantos en la fisiopatología y manejo de la patología endocrina más prevalente.

El comité científico convocó a la presentación de resúmenes de trabajos de investigación y reporte de casos clínicos, de los cuales fueron seleccionados 18 , en base a su diseño y relevancia en el manejo de la patología endocrina en nuestro medio.

Seis de estos trabajos de investigación fueron elegidos para su presentación oral durante el desarrollo del congreso. Fue premiado como el mejor, el Resumen \#14015: “Control metabólico en cohorte de pacientes diabéticos atendidos en el Programa de Diabetes del Hospital Nacional Dos de Mayo entre 2011 y 2013", presentado por Harold Torres y colaboradores.
Estos resúmenes son inéditos, por lo que agradezco en nombre del Consejo Directivo de la Sociedad Peruana de Endocrinologia y en el mío propio, la gentileza del Decano de la Facultad de Medicina de San Fernando, Dr. Herman Vildózola Gonzáles, y del Editor de Anales de la Facultad de Medicina, Dr. José Pacheco Romero, por brindarnos un espacio en el presente número de esta prestigiada revista científica, para su divulgación y cumplir así con nuestros fines académicos.

Dr. Jaime Villena

Presidente de la Sociedad Peruana de Endocrinologia

doi: http://dx.doi.org/10.15381/anales.v75i3.9789

\section{REFERENCIAS BIBLIOGRÁFICAS}

1. Garfias Dávila M. La formación de la universidad moderna en el Perú San Marcos 1850-1919. Lima, Perú: Asamblea Nacional de Rectores; 2010. 204 p.

2. Quinto Congreso Médico Latino Americano (Sexto Pan Americano). Actas y Trabajos. Lima: Libreria e Imprenta Sanmarti y Co.; 1914.

3. Almeida Md. The creation of chairs in tropical medicine in Peru and Brazil. História, Ciências, Saúde-Manguinhos. 2011;18(2):355-76.

4. Tejada VA. Historia del Instituto de Medicina Tropical "Daniel A. Carrión" de La Facultad de Medicina de San Fernando, de La Universidad Nacional Mayor de San Marcos. Rev Per Medicina Tropical. 2004;9(1):101-3. 



\title{
Frecuencia y factores asociados a la hipoglicemia en pacientes con diabetes mellitus tipo 2 hospitalizados en los servicios de medicina de un hospital general de Lima, Perú
}

\author{
Ray Ticse Aguirre \\ 'Servicio de Endocrinologia, Hospital Nacional Cayetano Heredia, Lima, Perú.
}

\begin{abstract}
Objetivo: Determinar la frecuencia de hipoglicemia en pacientes con diabetes mellitus tipo 2 (DM-2) hospitalizados y su asociación con los esquemas de insulinoterapia.

Metogología: Estudio descriptivo y transversal realizado durante los meses de julio a diciembre del 2013 en los servicios de Medicina del Hospital Nacional Cayetano Heredia (HNCH) de Lima-Perú. Se tomó información sobre edad, sexo, tiempo de enfermedad, esquemas de tratamiento previos a la hospitalización, durante la hospitalización, comorbilidades, complicaciones crónicas y diagnósticos de ingreso.
\end{abstract}

Los criterios de inclusión fueron: pacientes mayores de 18 años con diagnóstico de DM-2. Se excluyó los pacientes sometidos a intervenciones quirúrgicas, o si recibieron insulina endovenosa. Se definió hipoglicemia a valores de glicemia $\leq 70 \mathrm{mg} / \mathrm{dL}$. En los esquemas de insulinoterapia solo se consideró el uso de insulina NPH (Neutral Protamine Hagedorn) y/o insulina regular para los bolos de corrección o preprandiales. Las variables cuantitativas que no tenían distribución normal, fueron presentadas con medianas e intervalos intercuartil (IQT). Se consideró intervalos de confianza de $95 \%$ (IC) y valores significativos a valores de $p<0,05$. El proyecto fue aprobado por el comité de ética de la Universidad Peruana Cayetano Heredia.

Resultados: Ingresaron 159 pacientes con DM-2, de los cuales $136(85,5 \%)$ tuvieron el diagnóstico previo a la hospitalización y 23 $(14,5 \%)$ durante la hospitalización. Ingresaron 79 mujeres y 80 varones. La mediana de las edades fue 64 años (IQT: 55 a 74 ), la mediana del tiempo de enfermedad fue 7 años (IQT: 2 a 15); 54 (34\%) pacientes tuvieron tiempo de diagnóstico de DM-2 $\geq 10$ años.

La mediana de la glicemia de ingreso fue $174 \mathrm{mg} / \mathrm{dL}$ (IQT: 151 a 256). El $72 \%$ de los pacientes contó con valores de HbA1C, siendo la mediana 8,6\% (IQT: 6,7 a 9,7). 109 pacientes presentaron comorbilidades: insuficiencia renal (34\%), enfermedad cerebrovascular $(7,6 \%)$, hipertensión arterial $(51,6 \%)$, insuficiencia cardiaca congestiva $(2,5 \%)$ e insuficiencia hepática $(1,3 \%)$. La frecuencia de hipoglicemia fue $25,2 \%$.

Respecto a los esquemas de tratamiento, el más utilizado fue el esquema de insulina basal $(33,3 \%)$, seguido de basal y bolos preprandiales (31,5\%), basal y bolos de corrección (19,5\%), y solo bolos de corrección (15,7\%).

En el análisis de regresión logística, solo dos factores tuvieron relación significativa con episodios de hipoglicemia: uso del esquema de insulinoterapia basal y bolos preprandiales (OR: 3,43; IC: 1,62 a 7,25; $p=0,001)$, y tiempo de diagnóstico de DM-2 $\geq 10$ años (OR: $2,47 ; \mathrm{IC}: 1,18$ a 5,$16 ; \mathrm{p}=0,016)$.

Conclusiones: La frecuencia de hipoglicemia fue de $25,2 \%$ y los factores asociados fueron tiempo de enfermedad $\geq 10$ años y el uso del esquema de insulina basal NPH y bolos preprandiales de insulina regular. 


\title{
Variación de las concentraciones de parathormona y vitamina D en mujeres posmenopáusicas
}

\author{
Arquímedes Hidalgo ${ }^{1,2}$, Gerardo Ronceros ${ }^{1,2}$, Gustavo Cerrillo ${ }^{1,2}$, Oscar Ruiz ${ }^{1,2}$, \\ Fausto Garmendia ${ }^{1}$, Janet Villegas ${ }^{2}$, Ángela Chuquihuara ${ }^{2}$ \\ 'Instituto de Investigaciones Clínicas, Facultad de Medicina, Universidad Nacional Mayor de San Marcos. \\ ${ }^{2}$ Hospital Nacional Dos de Mayo.
}

Objetivo: Establecer la relación entre la concentración de parathormona (PTH) y vitamina D con la densitometria ósea en mujeres posmenopáusicas con puntaje $\mathrm{T} \leq-2,5$.

Metodología: Estudio observacional, transversal en 34 mujeres posmenopáusicas con puntaje $T \leq-2,5$ de 44 a 79 años, promedio 58,2, a quienes se midió la densitometría ósea en el antebrazo, columna vertebral, cadera, cuello femoral y trocánter, así como en sangre $\mathrm{Ca}, \mathrm{P}, \mathrm{PTH}$, vit D25, osteocalcina, proteínas plasmáticas, Hb, transaminasas, creatinina y, en orina, calcio, fósforo y NTX.

Resultados: Se encontró correlaciones entre edad y tiempo de menopausia con disminución de la densidad ósea medida en el antebrazo, cadera, cuello y trocánter femorales, no asi con la columna vertebral. Si bien con la edad incrementaron las concentraciones de PTH y disminuyeron las de vitamina D, las correlaciones no fueron estadisticamente significativas.

Conclusiones: La disminución de la densidad ósea vinculada a la edad y tiempo de menopausia no está relacionada a incremento de PTH ni a disminución de vitamina D.

Financiamiento: Trabajo realizado con financiamiento de la Fundación Instituto Hipólito Unanue. 


\title{
Asociación entre niveles séricos de hormonas tiroideas y concentración de lipoproteínas en mujeres de edad media con sintomatología crónica
}

\author{
Mirella Guarnizo Poma1', Socorro Paico Palacios ${ }^{1}$, Betzi Pantoja Torres ${ }^{1}$, Hosthen \\ Lenti Amorós ${ }^{1}$, Yony Miranda Tapia ${ }^{1}$, Laritza Montalvo Pastó ${ }^{1}$, Herbert Lázaro \\ Alcántara', Vicente Benites Zapata ${ }^{1}$, Adrián Hernández Díaz \\ Instituto Médico de la Mujer/Instituto Médico Metabólico, Lima, Perú.
}

\begin{abstract}
La relación entre deficiencia de hormonas tiroides y enfermedad ateroesclerótica ha sido descrita en muchos estudios. El riesgo cardiovascular asociado a hipotiroidismo está presente especialmente en mujeres.

Objetivo: Evaluar la relación entre triyodotironina libre (T3L), tiroxina libre (T4L) y hormona estimulante de tiroides (TSH) con los niveles de lipoproteínas de baja densidad (LDL) y lipoproteinas de alta densidad (HDL), en mujeres en edad media con sintomatología crónica.

Metodología: Estudio transversal, analítico y retrospectivo. Se revisó 218 historias clínicas seleccionadas al azar de mujeres que acudieron con síntomas ginecológicos crónicos y recurrentes (candidiasis/vaginosis recurrente, alteraciones en el patrón de menstruación, dolor pélvico crónico sin causa aparente, cambios del ánimo premenstrual, bochornos) a consulta externa de un centro de ginecología, entre 2007 y 2013. De ellas, 155 tuvieron los datos completos de LDL y hormonas tiroideas y 163 los datos completos de HDL y hormonas tiroideas. Las pacientes no tuvieron diagnósticos previos de enfermedades endocrinas o metabólicas. Se recolectó además edad, insulina basal (IB), cortisol salival en la mañana (CSAM) y porcentaje de grasa corporal (PGC) mediante bioimpedancia. Las mediciones de los valores recogidos de T3L, T4L, TSH, HDL y LDL tuvieron un intervalo de tiempo menor a 30 dias entre ellas. Para valorar las relaciones entre las variables usamos el coeficiente correlación de Pearson. Utilizamos el modelo de regresión lineal univariada y multivariada para evaluar la asociación entre hormonas tiroideas y los niveles de LDL y HDL.

Resultados: La media de edad fue 48,5 años $\pm 12,6$, las medias de T3L y T4L fueron 3,2 $\pm 0,4$ y 1,1 $\pm 0,2$, respectivamente; TSH presentó una mediana de 2,79 (RIC 1,96 a 3,95). Las medias de LDL y HDL fueron $137,7 \pm 37,7$ y $53,5 \pm 15,4$, respectivamente. T3L y LDL estuvieron inversamente correlacionadas $(r=-0,23, p<0,01)$ y $T 4$ y HDL estuvieron directamente correlacionadas $(r=0,15, p=0,05)$. La regresión lineal univariada mostró que por cada aumento de una unidad de T3L el LDL disminuyó en -17,6 ( $p<0,01)$; además, mostró que por cada aumento de una unidad de T4L el HDL aumentó en 14,4 ( $p=0,05)$. Después de ajustar por edad, IB, CSAM y PGC la asociación entre T3L y LDL se mantuvo estadísticamente significativa: por cada unidad que aumentaba T3L, el LDL disminuyó $-23,8$ $(p=0,02)$. No hubo asociación entre TSH y las lipoproteinas; de igual modo no encontramos asociación entre T3L y HDL o T4L y LDL.

Conclusiones: Encontramos una relación inversa entre T3L y el LDL en mujeres adultas con síntomas crónicos sin antecedentes previos de enfermedad endocrina. Mantener niveles normales de T3L puede contribuir a mantener el LDL por debajo de rangos patológicos
\end{abstract}




\title{
Características clínico-laboratoriales de pacientes con parálisis periódica tirotóxica en el Hospital Nacional Edgardo Rebagliati Martins, 2011-2014
}

\author{
Sofía Sáenz-Bustamante Sofía ${ }^{1 a}$, Marlon Yovera-Aldana ${ }^{2 a}$, María Churampi-López ${ }^{1 a}$, \\ Natali Jáuregui-Macedo ${ }^{1 \mathrm{~b}}$, Marialejandra Delgado-Rojas ${ }^{1 \mathrm{~b}}$, David Liviac-Cabrera ${ }^{1 \mathrm{~b}}$, \\ Vitalia Ranilla-Seguín ${ }^{1 \mathrm{~b}}$, José Paz-Ibarra ${ }^{1 \mathrm{a}}$ \\ ${ }^{1}$ Hospital Nacional Edgardo Rebagliati Martins. ${ }^{2}$ Hospital Departamental de Huancavelica, MINSA. \\ ${ }^{a}$ Médico Endocrinólogo. ${ }^{\mathrm{b}}$ Residente de Endocrinología.
}

Introducción: La parálisis periódica tirotóxica (PPT) es la causa más común de parálisis periódica hipokalémica (PPH) adquirida. Es frecuentemente transitoria, pero potencialmente letal. Debe tenerse en cuenta en el diagnóstico diferencial de todo paciente con parálisis flácida aguda (PFA), para un adecuado manejo terapéutico a corto y largo plazo.

Objetivo: Describir las características clínicas y laboratoriales de pacientes con PPHT del HNERM, periodo 2011-2014.

Metodología: De los pacientes con hipertiroidismo atendidos en el Servicio de Endocrinología y Metabolismo del HNERM, en el periodo julio 2011 a junio 2014, se seleccionó a aquellos que presentaron la triada PFA, tirotoxicosis e hipokalemia durante las crisis paralíticas. El diseño de estudio fue descriptivo, transversal, retrospectivo, mediante revisión de historias clínicas. Se describió las características clínicas y paraclínicas de los pacientes.

Resultados: De 410 pacientes con hipertiroidismo, se detectó 8 pacientes con PPT. La media de edad al diagnóstico fue 38,4 años (rango: 31 a 47 años); el 100\% fue varón, 100\% de raza mestiza, 12,5\% con antecesor asiático, 25\% nació en departamentos de conocida deficiencia de yodo y $50 \%$ tenía antecedente familiar de enfermedad tiroidea. Como factores precipitantes, el $75 \%$ tuvo el alimentario (consumo copioso de alimentos hipercalóricos o alto contenido de sal o exceso de bebidas azucaradas) y $50 \%$ tuvo el físico (actividad física extenuante o caminatas). El 75\% de PPT no fue diagnosticado previamente de hipertiroidismo, es decir, se diagnosticó hipertiroidismo con el episodio de PPHT; sin embargo, presentaban un promedio de tiempo de presencia de signos y sintomas hipertiroideos de 7 meses (rango: 0 a 24 m). El otro 25\% tuvo diagnóstico de hipertiroidismo antes del primer episodio de PPT, con un tiempo medio de 1,5 meses (rango: 1 a 2 meses); solo un caso no presentó síntomas previos de hipertiroidismo antes del primer episodio de PPT. El patrón motor predominante fue la paraplejía (63\%), seguido de tetraparesia (25\%) y paraparesia (12\%); además, se presentó un caso con paro cardiorrespiratorio, sin consecuencias posteriores. El $50 \%$ de los casos presentó solo un episodio, $37,5 \%$ dos episodios y 12,5\% 3 episodios previos, con una media de duración de cada episodio de 2,7 horas (rango: 0,5 a 8 horas); el $50 \%$ se presentó en las primeras horas de la mañana y el resto durante la noche. La kalemia promedio fue 2,5 mEq/L (rango: 1,4 a $3,5 \mathrm{mEq} / \mathrm{L}$ ). Finalmente, la etiología de la tirotoxicosis fue en $87,5 \%$ debida a enfermedad de Graves y su intensidad fue severa en $62,5 \%$, moderada en $25 \%$ y leve en $12,5 \%$, según escala Pohl.

Discusión: La PPH podria estar relacionada a mutaciones en el canal de potasio kir 2,6. Es una entidad patológica que precisa de un rápido diagnóstico, pues aunque es reversible, amerita tratamiento urgente por el riesgo de mortalidad asociada a complicaciones cardiacas. Debe sospecharse PPH en todo paciente varón joven que presente debilidad muscular aguda, aún cuando no exista sintomatología o signologia de hipertiroidismo o etnia caracteristica.

Conclusiones: Dos de cada 100 pacientes hipertiroideos presentaron PPT. Ocurrió en varones entre 30 y 50 años, con un promedio de 7 meses de inicio de signos y sintomas de hipertiroidismo, siendo estos mayormente de intensidad severa, presentándose predominantemente como una paraplejia de menos de 8 horas de duración. 
1405

\title{
Asociación entre disglicemia inicial y disglicemia por prueba de tolerancia oral a glucosa al séptimo día en sujetos postinfarto de miocardio agudo del Hospital Nacional Edgardo Rebagliati Martins, durante el periodo mayo a julio 2014
}

\author{
María Churampi López ${ }^{1 \mathrm{a}}$, Marlon Yovera Aldana ${ }^{2 \mathrm{a}}$, Natali Jáuregui Macedo ${ }^{1 \mathrm{~b}}$, Sofía \\ Sáenz Bustamante ${ }^{1 \mathrm{~b}}$, Marialejandra Delgado Rojas ${ }^{1 \mathrm{~b}}$, David Liviac Cabrera $^{1 \mathrm{~b}}$ \\ ${ }^{1}$ Hospital Nacional Edgardo Rebagliati Martins. ${ }^{2}$ Hospital Departamental de Huancavelica, MINSA \\ ${ }^{a}$ Médico Endocrinólogo. ${ }^{b}$ Residente de Endocrinología.
}

\begin{abstract}
La diabetes mellitus tipo 2 no diagnosticada es algo frecuente, reportándose un retraso en el diagnóstico entre 5 y 7 años. Las personas con intolerancia a la glucosa y DM2 no diagnosticadas tienen un riesgo incrementado de enfermedad coronaria, ictus y enfermedad vascular periférica. Se ha estudiado la asociación entre disglicemia inicial posterior a infarto de miocardio agudo y desarrollo de diabetes. El desempeño de la PTOG al $7^{\circ}$ día es reportado como similar al método convencional ambulatorio realizado al tercer, sexto, noveno y décimo segundo mes postevento.

Objetivo: El presente estudio describió la asociación entre un valor glicémico alterado post IMA y disglicemia por PTOG realizada al séptimo día.

Metodología: Los pacientes fueron seleccionados al ingreso a la Unidad de Cuidados Coronarios del Hospital Edgardo Rebagliati Martins durante los meses de mayo a julio de 2014. El diseño de estudio fue un descriptivo, analítico a 2 grupos, longitudinal, prospectivo. Se le realizó glucosa al ingreso; al cuarto día de hospitalización, hemoglobina glicosilada; y al sétimo día de hospitalización, la prueba de tolerancia a la glucosa con glucosa anhidra $75 \mathrm{~g}$, luego de un periodo de ayuno de 8 horas. Para realizar la PTOG, el paciente estuvo hemodinámicamente estable.

Resultados: Se estudió un total de 19 pacientes. El promedio de glucosa al ingreso fue $133 \pm 44 \mathrm{mg} / \mathrm{dL}$, la glicemia al $4^{\circ}$ día fue 122 $\pm 31 \mathrm{mg} / \mathrm{dL}$; en la PTOG al $7^{\circ}$ día, la media de glucosa basal fue $115 \pm 22 \mathrm{mg} / \mathrm{dL}$ y la posprandial 2 horas fue $159 \pm 58 \mathrm{mg} / \mathrm{dL}$. La media de Hba1c fue $6,5 \pm 1,2 \%$. El $88 \%$ de las PTOG fue anormal (26\% glucosa alterada en ayunas, $21 \%$ intolerancia a la glucosa, $32 \%$ diabetes mellitus). La glucosa al ingreso del grupo PTOG anormal fue $140 \mathrm{mg} / \mathrm{dL}$ y el de PTOG normal fue $106 \mathrm{mg} / \mathrm{dL}$ ( $p=0,53$ Wilcoxon). Sin embargo, se encontró asociación entre glucosa al cuarto dia y PTOG anormal ( $p=0,02$ Wilcoxon), como también con hemoglobina glicosilada ( $p=0,01$ Wilcoxon).
\end{abstract}

Conclusiones: No se encontró asociación en los pacientes que tienen una disglicemia inicial post IMA, con PTOG anormal realizada el séptimo día. Sí lo hubo con glicemia al cuarto día y hemoglobina glicosilada. 


\title{
Tiroiditis fibrosante de Riedel. A propósito de un caso
}

\author{
David Liviac Cabrera", José Paz Ibarra", Marialejandra Delgado Rojas", \\ Natali Jáuregui Macedo ${ }^{1}$, Sofía Sáenz Bustamante" ${ }^{1}$, José Somocurcio \\ Peralta $^{2}$, Vitalia Ranilla Seguín ${ }^{1}$ \\ Servicio de Endocrinologia y Metabolismo, Hospital Edgardo Rebagliati Martins. \\ ${ }^{2}$ Departamento de Anatomía Patológica, Hospital Edgardo Rebagliati Martins.
}

Objetivo: Presentar un caso de tiroiditis de Riedel.

Metodología: Se presenta las características clínicas y paraclínicas de una paciente con tiroiditis de Riedel.

Resultados: Mujer de 68 años acudió al Consultorio de Endocrinología, referida, para estudio de nódulo tiroideo hallado durante examen clínico rutinario. Tenía antecedente de hipotiroidismo diagnosticado a los 28 años, que recibió tratamiento sustitutivo por 20 años. Ecografía tiroidea: imagen de aspecto sólido heterogéneo de 22x20 mm en LTI, que requiere mayor estudio. BAAF: Tumor folicular. TSH: $3,97 \mathrm{uUI} / \mathrm{mL}$. T4L: $1 \mathrm{ng} / \mathrm{dL}$. AbTPO: $38,8 \mathrm{UI} / \mathrm{mL}$. Fue sometida a tiroidectomía, cuya evaluación patológica concluyó: Bocio adenomatoso con degeneración quistica a nivel de LTI. Tiroiditis crónica inespecífica en istmo y LTD. En el seguimiento postoperatorio requirió $200 \mathrm{ug} / \mathrm{d}$ de LT4 durante los cuatro primeros años. Posteriormente, precisó disminución de dosis por tirotoxicosis facticia. Posteriormente, por síntomas compresivos en cuello y bocio irregular aumentado de consistencia, se realizó ecografía que evidenció ausencia de LTD y presencia de LTI aumentado de volumen, de parénquima heterogéneo, con presencia de formación sólida heterogénea de bordes mal definidos de aproximadamente $23 \mathrm{~mm}$ de diámetro, proyectado a nivel posteroizquierdo del lóbulo y adyacente a estructura vascular yugular y carotídea, asociado a adenopatías en grupo III izquierdo y II derecho, con diferenciación corticomedular conservada. BAAF de nódulo izquierdo: Bocio coloide en 2 oportunidades. Por persistencia de disconfórt cervical y síntomas compresivos, se decidió tiroidectomía complementaria, cuya evaluación patológica informó: Glándula tiroides reemplazada en su totalidad por tejido conectivo denso que infiltra planos musculares, asociado a presencia de pigmento de hemosiderina y proceso inflamatorio crónico perivascular. Los hallazgos morfológicos corresponden a tiroiditis fibrosante de Riedel. Tg post tiroidectomía $<0,2$ $\mathrm{ng} / \mathrm{mL} ; \mathrm{AbTg}<20 \mathrm{Ul} / \mathrm{mL}$. Ecografía post tiroidectomía: No imágenes definidas de tejido glandular ni recidiva de enfermedad. TEM de órbitas y tóraco abdominal: normal.

Discusión y conclusiones: La tiroiditis de Riedel es una enfermedad inflamatoria crónica rara caracterizada por invasión de la glándula tiroidea y estructuras adyacentes. Puede estar relacionada a otros procesos inflamatorios fibrosantes en mediastino, retroperitoneo, pseudotumor orbitario y colangitis esclerosante 0 aparecer de forma aislada. Se reporta en $0,05 \%$ de las cirugias tiroideas y suele ser más frecuente en mujeres de mediana edad. Su etiología aún no ha sido esclarecida, pero se postula que podría ser el estadio final de la enfermedad de Hashimoto, un desorden vascular del colágeno o una enfermedad autoinmune. La clínica es inespecifica y puede simular un carcinoma invasivo (bocio no doloroso que crece progresivamente o de forma súbita y que puede producir sintomas compresivos). El diagnóstico por BAAF no es posible debido a la densa fibrosis que no permite la toma de una muestra adecuada ni realizar el diagnóstico diferencial con un carcinoma indiferenciado. La cirugía confirma el diagnóstico, excluyendo malignidad, y permitiendo aliviar los sintomas compresivos. 


\title{
Mujer portadora de asociación de carcinoma papilar y medular de tiroides
}

\author{
David Liviac Cabrera', José Paz Ibarra', Marialejandra Delgado Rojas", \\ Natali Jáuregui Macedo", Vitalia Ranilla Seguín', Sofía Sáenz Bustamante", \\ José Somocurcio Peralta ${ }^{2}$ \\ ${ }^{1}$ Servicio de Endocrinologia y Metabolismo, Hospital Edgardo Rebagliati Martins. \\ ${ }^{2}$ Departamento de Anatomia Patológica, Hospital Edgardo Rebagliati Martins.
}

\begin{abstract}
Objetivo: Presentar un caso infrecuente de asociación de carcinoma papilar (CPT) y carcinoma medular de tiroides (CMT). Metodología: Se presenta las características clínicas y paraclínicas de una paciente con asociación de CPT y CMT.

Resultados: Mujer de 58 años acudió al Consultorio de Endocrinología, con un tiempo de enfermedad de 12 meses, refiriendo aumento de volumen cervical anterior, asociado a disfagia, disfonía y odinofagia. Antecedentes familiares de cáncer de tiroides, sin precisar la variedad (hermana), y cáncer cervical, sin precisar localización (padre). Analítica: T4 1,30 ng/dL, TSH 1,66 uUl/ml, AbTPO (-), calcitonina 13,8 pg/mL. Ecografía de tiroides: LTD 42,2×16,2×13,3 mm con nódulo hipoecoico de 10,7×9,6 mm en tercio medio. LTI $31,7 \times 10,2 \times 10,4 \mathrm{~mm}$ disminuido de volumen, de ecogenicidad levemente heterogénea, con nódulo calcificado de 5,7×4,6 $\mathrm{mm}$ en polo superior. Istmo de $2,4 \mathrm{~mm}$ de espesor. BAAF de tumoración: CPT. Fue sometida a tiroidectomía total en clínica particular, cuya evaluación patológica informó: CPT variante clásica con calcificación distrófica de $8 \mathrm{~mm}$ de diámetro aproximado, parcialmente encapsulado, ubicado en el LTD; ausencia de embolia vascular, linfática y perineural, sin infiltración de cápsula tiroidea; quiste coloide ubicado en polo inferior de LTD. CMT de 7×5 mm, patrón nodular. IHQ: TTF-1 (-), TG (-), calcitonina (+) en LTI. Istmo libre de neoplasia maligna.

Discusión y conclusiones: CPT es el tipo más común de los cánceres de tiroides, constituyendo 70\% a 80\% de todos los casos. Tiende a crecer lentamente y a extenderse primeramente a los ganglios linfáticos del cuello. Presenta un excelente pronóstico. Por otro lado, el CMT es responsable de $5 \%$ a $10 \%$ de todos los cánceres de tiroides, y suele formar parte del Sd. de NEM 2. Es de peor pronóstico, dado su gran potencial de metástasis hematógena. Recientes estudios han revelado que el proto-oncogén RET estaría envuelto en la oncogénesis de ambos carcinomas, por la activación de la enzima tirosinaquinasa, ya sea por mutaciones puntuales de su receptor (NEM2 asociado a CMT) o por reordenamiento del gen (CPT). Su asociación no es común, describiéndose en la literatura inglesa un aproximado de 20 casos.
\end{abstract}




\title{
Mujer de 32 años con manifestaciones severas de hipercortisolismo por enfermedad de Cushing cíclico
}

\author{
Marialejandra Delgado", José Paz-Ibarra', Sofía Sáenz ${ }^{1}$, Natalia Jáuregui ${ }^{1}$, \\ David Liviac ${ }^{2}$, Vitalia Ranilla', Oscar Quintana ${ }^{1}$ \\ ${ }^{1}$ Servicio de Endocrinología, Hospital Edgardo Rebagliati, Lima, Perú.
}

Objetivo: Presentar un caso de hipercortisolismo cíclico en enfermedad de Cushing (EC).

Método: Se presenta las características clínicas y paraclínicas de un paciente con EC con secreción cíclica de ACTH.

Resultados: Mujer de 32 años, procedente de Lima, con dos años de diagnóstico de síndrome de ovario poliquístico (SOPQ), por lo que recibió anticonceptivos orales (ACOs) por un año. Dos meses antes del ingreso tuvo diagnóstico de diabetes mellitus (DM) de difícil control, por lo que recibió tratamiento con MTF e insulina. Tres semanas antes del ingreso hubo alteración del comportamiento, realizaba actividades que luego no recordaba, palpitaciones, sensación de calor y debilidad marcada de extremidades. El día de ingreso por emergencia presentó agitación psicomotriz, trastorno del sensorio, hiperglicemia e hipokalemia severa, por lo que fue hospitalizada. Al examen: lúcida, alerta, ventilaba espontáneamente, facies cushingoide. Peso $60 \mathrm{~kg}$, IMC 23, PA 120/60 mmHg, FC 92x', FR 16x'. Piel: hirsutismo leve-moderado, múltiples equimosis, no estrías. TCSC: pequeños cojinetes en fosa supraclavicular, pequeña giba dorsal, edema ++/+++ en miembros inferiores. Analítica: G $459 \mathrm{mg} / \mathrm{dL}, \mathrm{Na}+149 \mathrm{mEq} / \mathrm{L}, \mathrm{K}+1.99 \mathrm{mEq} / \mathrm{L}$, cortisol 8 am $>50 \mathrm{ug} / \mathrm{dL}$, ACTH $386 \mathrm{pg} / \mathrm{mL}$, cortisol $00 \mathrm{~h}>50 \mathrm{ug} / \mathrm{dL} ; \mathrm{TSH} 1,54 \mathrm{uUl} / \mathrm{mL}, \mathrm{T} 4 \mathrm{~L}$ 0,54 ng/dL; T3L 1,00 pg/mL, péptido C 0,27 ng/mL, estradiol 29,0 pg/mL, Pg 0,80 ng/mL, LH 0,10 mlU/mL, PRL 7,25 ng/mL, androstenediona >10 ng/mL, DHEAS $265 \mathrm{ug} / \mathrm{mL}$, testosterona libre 3,20 pg/mL. Pruebas funcionales: CLU basal 1: $919,35 \mathrm{ug} / 24 \mathrm{~h}$, CLU basal 2: 1592,5 ug/24 h, CLU post Dexa8 $132 \mathrm{ug} / \mathrm{dL}$. Cortisol sérico post Dexa8 >50 ug/dL. Infusión EV de Dexa7mg (cortisol basal 90,30 ug/dL, cortisol 4 pm 13,5 ug/dL, cortisol 24 h post 98,10 ug/dL. RMN: imagen nodular de $7 \mathrm{~mm}$ en lado derecho de la hipófisis; TEM TAP normal. Ante la sospecha de Sd. de Cushing (SC) ectópico, se indicó CSPI, el cual no se realizó por causas no médicas. Fue sometida a cateterismo de venas yugulares internas: ACTH central $100 \mathrm{pg} / \mathrm{mL}$ y ACTH periférico $60 \mathrm{pg} / \mathrm{mL}$, relación ACTH central/periférico 1,6. En espera de intervención quirúrgica (4 semanas), se ha tomado controles, ACTH 44,60 pg/mL, androstenediona 2,07 ng/mL, DHEAS 32,60 ug/dL, cortisol 18,6 ug/dL.

Discusión: La EC es la causa más común de SC espontáneo, en 60 a 70\%; es el resultado de la hipersecreción de ACTH por un adenoma hipofisario. El SC cíclico es un patrón de hipercortisolismo, en el que la bioquímica de la producción de cortisol fluctúa rítmicamente y retrasa el diagnóstico. Ante la sospecha clínica de un SC, debemos demostrar la presencia de hipercortisolismo, la ruptura del ritmo circadiano y la falla en la supresión a corticoide exógeno. En nuestra paciente evidenciamos niveles aumentados de CLU y cortisol plasmático, la supresión de CLU >90\% post Dexa8, supresión temprana de cortisol plasmático en la infusión de Dexa7mg y cateterismo de VYI con una relación ACTH central/ periférica $>1,6(\mathrm{~S}: 81 \%)$ que nos orienta a EC.

Conclusión: El SC cíclico debe ser sospechado en pacientes con sintomas o signos de SC, pero con los niveles normales de cortisol, 0 con la fluctuación de los valores de cortisol o respuestas anómalas a la dexametasona, recomendándose la realización de las pruebas dinámicas durante la actividad de la enfermedad y tener un alto índice de sospecha que contemple un seguimiento a largo plazo. 


\title{
Síndrome de Doeger Potter, un caso inusual de hipoglicemia recurrente
}

\author{
Marialejandra Delgado ${ }^{1}$, José Paz-lbarra', Sofía Sáenz¹, Natalia Jáuregui', \\ David Liviac², Vitalia Ranilla', Oscar Quintana ${ }^{1}$ \\ ${ }^{1}$ Servicio de Endocrinología, Hospital Edgardo Rebagliati, Lima, Perú.
}

Objetivo: Presentar un caso de hipoglicemia paraneoplásica recurrente.

Método: Se presenta las características clínicas y paraclínicas de un paciente con hipoglicemia recurrente, como manifestación de un tumor fibroso solitario de pleura (TFSP) recidivante conocido como síndrome de Doege Potter (SDP).

Resultados: Varón de 66 años con antecedente de neumonectomía izquierda por tumor fibroso solitario gigante, en INEN, en 2006. Procedente de Ica, refirió que 10 meses antes de su ingreso presentó dolor tipo opresivo en hipocondrio y flanco izquierdos asociado a aumento de volumen en dichas regiones, disnea de esfuerzo y sensación de llenura precoz, asociados a pérdida ponderal de 5 kg en 6 meses; además, refería presencia de episodios de sudoración profusa, seguido de confusión y conducta anormal, que se presentaban en la madrugada y que mejoraban con la ingesta de bebidas o alimentos azucarados. Ingresó a emergencia con trastorno de conciencia asociado a glicemia de $31 \mathrm{mg} \%$. Al examen estaba lúcido, alerta, taquipneico. Peso $60 \mathrm{~kg}, \mathrm{IMC}$ 22; PA 110/60 mmHg, FC 82x', FR 28x', facies acromegaloide, cicatriz de toracotomía izquierda, MV abolido en HTI; masa palpable en hipocondrio y flanco izquierdos que llegaba hasta mesogastrio, edema de miembros inferiores, fóvea (+). Analítica: $\mathrm{G}$ ayunas 31 a $60 \mathrm{mg} / \mathrm{dL}$, insulinemia ayunas <2,00 ulU/mL; péptido C ayunas <0,10 ng/mL. IGF-1: $100 \mathrm{ng} / \mathrm{mL}$ (VN: 69 a 200); IGF-2: $398 \mathrm{ng} / \mathrm{mL}$ (VN: 50-250). Hormonas contra-reguladoras normales. TEM TAP: Gran masa solida heterogénea de contornos definidos de aspecto sarcomatoso que ocupa la base del hemitórax izquierdo e hipocondrio y flanco del mismo lado, de 19×18×32 cm de diámetro AP, DT y DL; desplaza posteriormente al bazo y lateralmente a las asas intestinales; asi mismo, ejerce efecto de desplazamiento del páncreas y riñón izquierdo.

Discusión: La hipoglucemia inducida por tumor es una rara entidad que puede ocurrir en pacientes con diversos tipos de tumores y que puede ser causada por diferentes mecanismos: secreción de insulina por islotes pancreáticos (insulinoma), secreción ectópica de insulina por TNEs y GISTs. Además, hay casos de hipoglicemia inducida por tumores no pancreáticos, donde la principal etiología de la hipoglucemia es producción de IGF2 o su precursora de alto peso molecular (grande IGF2). Otros mecanismos son secreción de IGF-1, producción de auto-anticuerpos contra la insulina o su receptor o, más raramente, la secreción de GLP1. En el SDP, se ha postulado varias teorías sobre los factores responsables de la hipoglicemia: secreción excesiva por el tumor de IGF-2, falla en los mecanismos compensadores para prevenir la hipoglicemia y acelerado consumo de glucosa por tumores de gran tamaño.

Conclusiones: Ante la ocurrencia de hipoglicemia sintomática repetida (sin relación a causas como DM, uso de hipoglicemiantes orales u otras endocrinopatías), debe descartase un síndrome para-neoplásico tipo SDP. LoS TFSP pueden manifestarse clínicamente con hipoglicemia sintomática. La resección quirúrgica es el tratamiento de elección en estos pacientes. 


\title{
Carcinoma pobremente diferenciado de tiroides. A propósito de un caso
}

\author{
David Liviac Cabrera', José Paz Ibarra', Marialejandra Delgado Rojas, \\ Natali Jáuregui Macedo ${ }^{1}$, Vitalia Ranilla Seguín ${ }^{1}$, Sofía Sáenz Bustamante ${ }^{1}$, \\ José Somocurcio Peralta ${ }^{2}$ \\ ${ }^{1}$ Servicio de Endocrinología y Metabolismo, Hospital Edgardo Rebagliati Martins. \\ ${ }^{2}$ Departamento de Anatomia Patológica, Hospital Edgardo Rebagliati Martins.
}

Objetivo: Presentar un caso de carcinoma pobremente diferenciado de tiroides (CPDT).

Metodología: Se presenta las características clínicas y paraclínicas de una paciente con CPDT.

Resultados: Mujer de 64 años que acude al Consultorio de Cirugía de Cabeza y Cuello, referida del Hospital de Ica, con un tiempo de enfermedad de 6 meses, refiriendo aumento de volumen cervical anterior progresivo asociado a sensación de opresión, no disfagia, no odinofagia, no disfonía; fue catalogado como un bocio grado III. Antecedentes familiares sin importancia. TEM cervical: amplia lesión compleja de aspecto neoformativo que protruye desde el lóbulo tiroideo derecho desplazando las estructuras circundantes, aparentemente sin infiltrarlas en forma significativa, aún cuando contacta extensamente con el sistema vascular, muscular y la vía aérea. No presenta signos de extensión intraluminal, solo las desplaza, incluyendo un significativo desplazamiento izquierdo de la tráquea con compresión de su luz. No se observa adenomegalias de volumen significativo. Incidentalmente, se objetiva pequeño nódulo subpleural/pleural en el nivel del lóbulo superior izquierdo, sin adenomegalias mediastinales significativas. TEM de tórax: Nódulo sólido bien definido, de base pleural, de $2 \mathrm{~cm}$, en segmento III pulmonar. BAAF de tumoración: Citología de tumor folicular con atipia. Fue sometida a tiroidectomía total, cuya evaluación patológica informó: CPDT de 90x60 mm, con áreas de aspecto predominantemente insular y sólido, otras áreas trabecular y tubular, con extensas áreas de necrosis; infiltra la cápsula y se extiende a los tejidos blandos adyacentes, con embolia vascular tumoral venosa, ubicado sobre el LTI. Tiroides remanente con áreas de hiperplasia folicular con degeneración quística y débil; hiperplasia papilar intrafolicular. Tiroiditis crónica leve. IHQ: TTF-1 (+), TG (+), Ki-67 >10\%. Es referida a Consultorio de Endocrinologia, donde se solicitó exámenes pre-ablativos: TSH $>75 \mu \mathrm{U} / \mathrm{mL}, \mathrm{Tg} 1,47 \mathrm{ng} / \mathrm{mL}$, $\mathrm{AbTg}(-) ;$ PTH 66,9 pg/mL.

Discusión y conclusiones: Los CPDT son tumores situados morfológica y biológicamente en una posición intermedia entre los bien diferenciados (papilar y folicular) y los totalmente indiferenciados o anaplásicos. Son poco frecuentes, siendo la variedad histológica insular la más común entre ellos y la de peor pronóstico si esta se encuentra en forma dominante. Este tipo histológico está caracterizado por la formación de islotes de células tumorales que contienen un número variable de pequeños folículos con tiroglobulina, células pequeñas y homogéneas en cuanto a su tamaño, actividad mitótica variable, invasión capsular y vascular, y frecuentes focos de necrosis. El tratamiento de elección es la tiroidectomia total y disección ganglionar del cuello, seguido de la ablación con radioyodo. No existen muchas series publicadas, pero la evolución suele ser desfavorable, a pesar del tratamiento, con numerosas recurrencias y fallecimientos. 


\title{
Paciente con carcinoma papilar asociado a carcinoma insular de tiroides metastásico
}

\author{
Natali Jáuregui Macedo", José Paz-Ibarra', Sofía Sáenz Bustamante', \\ David Liviac Cabrera', Marialejandra Delgado Rojas ${ }^{1}$, Vitalia Ranilla \\ Seguín ${ }^{1}$, José Somocurcio Peralta ${ }^{2}$ \\ ${ }^{1}$ Servicio de Endocrinología y Metabolismo, Hospital Edgardo Rebagliati Martins. \\ ${ }^{2}$ Departamento de Anatomía Patológica, Hospital Edgardo Rebagliati Martins.
}

\begin{abstract}
Objetivo: Presentar un caso de carcinoma insular de tiroides (CIT) asociado a carcinoma papilar de tiroides (CPT). Metodología: Se presenta las características clínicas y paraclínicas de una paciente con asociación de CIT y CPT.

Resultados: Mujer de 67 años, natural de Junín, procedente de Lima. Un año antes del ingreso inicia padecimiento con dolor e impotencia funcional en cadera derecha, no asociada a trauma, pero sí a tumor en cuero cabelludo de $2 \times 2 \mathrm{~cm}$. Diagnostican 'fractura patológica'. Se realiza biopsia del nódulo cuero cabelludo con resultado anatomopatológico (AP) de metástasis de primario desconocido. TEM corporal mostró imágenes metastásicas múltiples (pulmón, hígado, cerebral, renal, adrenal). Posteriormente, perdió contacto con los servicios de salud. Cinco meses después ingresó a emergencia por dolor lumbar y disfagia; se evidenció tiroides aumentada de tamaño. En la revisión de AP: Adenocarcinoma medianamente diferenciado metastásico de probable origen pulmonar

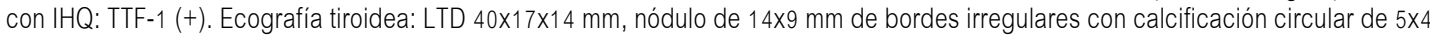
$\mathrm{mm}$ y nódulo hipoecogénico de 8x7 mm y otros nódulos $<5 \mathrm{~mm}$. LTI 57×31×42 mm, con nódulo de 43×27 mm heterogéneo, de bordes irregulares, parcialmente calcificado por otros nódulos $<8 \mathrm{~mm}$. Istmo de $7 \mathrm{~mm}$. BAAF sugestivo de carcinoma insular de tiroides; Tg $>300 \mathrm{ng} / \mathrm{dL}$, calcitonina $<2 \mathrm{pg} / \mathrm{mL}$. Recibió RTE holocraneal y en fémur derecho. La tiroidectomía total reveló tumor pétreo de $5 \times 4 \times 3 \mathrm{~cm}$ que ocupaba LTI e istmo y otro de $3 \times 2 \mathrm{~cm}$ en LTD; ganglio de $1 \times 0,5 \mathrm{~cm}$. AP: CPT variedad folicular $90 \%$ y clásica $10 \%$ infiltrante, de $1,2 \times 1 \mathrm{~cm}$, ubicado en LTD e istmo, con microembolia tumoral linfática que infiltra la cápsula y se extiende hacia los tejidos blandos. En LTI: Carcinoma pobremente indiferenciado de patrón insular, infiltra la cápsula, extendiéndose a tejido adyacente. Tiroides remanente con áreas de atrofia. Recibió $150 \mathrm{mCi}$ de radioyodo. Se realizó RCT posterapia, con resultado de captación positiva cuello.

Discusión y conclusiones: El cáncer de tiroides es la neoplasia maligna más frecuente del sistema endocrino. El CPT representa el $80 \%$ de todos los canceres de tiroides. En cambio, el CIT representa $<5 \%$. Ambos son más frecuentes en la mujer. A diferencia de los carcinomas diferenciados, la edad de presentación del CIT es más avanzada (54 vs. 36 a); en el momento del diagnóstico es mayor el tamaño del tumor, y más frecuente la existencia de metástasis (26 vs. 2\%). Se define histológicamente por la agrupación de células foliculares que forman islotes y que presentan habitualmente focos de necrosis central. Las células del CIT son uniformemente pequeñas, tanto en tamaño nuclear como citoplasmático, con nucléolos prominentes y cromatina finamente granular. Se encuentra mitosis escasas. El tratamiento del CIT es similar al de los CDT: cirugía, radioyodo y tratamiento supresivo con LT4. Cabe recalcar que tiene menor respuesta al radioyodo que el CPT. La constatación de que la mayoría de estos tumores, a pesar de su desdiferenciación, concentran radioyodo, tiene implicancias en el seguimiento y, además, apoyaría la indicación de esta terapia. Los pacientes que padecen un CIT presentan peor pronóstico que aquellos con CDT, debido a un mayor índice de recurrencia y a una mayor mortalidad.
\end{abstract}




\title{
Carcinoma adrenocortical funcionante. A propósito de un caso
}

\author{
Natali Jáuregui Macedo ${ }^{1}$, José Paz-lbarra', Sofía Sáenz Bustamante", \\ David Liviac Cabrera ${ }^{1}$, Marialejandra Delgado Rojas ${ }^{1}$, Vitalia Ranilla \\ Seguín ${ }^{1}$, Luis Velarde Ponce ${ }^{2}$, José Somocurcio Peralta ${ }^{3}$ \\ ${ }^{1}$ Servicio de Endocrinología y Metabolismo, Hospital Nacional Edgardo Rebagliati Martins. \\ ${ }^{2}$ Departamento de Urología, Hospital Nacional Edgardo Rebagliati Martins. \\ ${ }^{3}$ Departamento de Anatomía Patológica, Hospital Nacional Edgardo Rebagliati Martins.
}

Objetivo: Presentar un caso de carcinoma adrenocortical (CAC) funcionante.

Metodología: Se presenta las caracteristicas clínicas y para-clínicas de una paciente con CAC funcionante de años de evolución.

Resultados: Mujer de 64 años, procedente de Ica. Hermanos con HTA y DM2. Ausencia de desarrollo puberal y amenorrea primaria, con menarquia a los 22 años con el uso de anticonceptivos y prednisona por diagnóstico de 'hiperplasia suprarrenal congénita'. Fue sometida a clitoroplastia. Refiere que al diagnóstico le informaron la existencia de 'tumor suprarrenal' no filiado, sin seguimiento posterior y tratamiento hormonal irregular. GOPO; hace 2 años HTA de difícil control y crisis hipertensivas a repetición, y posterior diagnóstico de DM2 en enero de este año, que requiere insulinoterapia para control. Cuatro meses antes del ingreso inicia con edema progresivo de miembros inferiores (mmii), vértigos subjetivos y episodios de cefalea pulsátil en región occipital, sin flushing, asociados a incrementos marcados de PA, acudiendo a emergencia de forma recurrente por este motivo. Se añade disnea progresiva a medianos esfuerzos, palpitaciones, disminución de fuerza muscular en extremidades, por lo que acude a emergencia, encontrándose hipertensa, IMC 23,8 kg/m2, atrofia de piel, edema de extremidades con fóvea; múltiples equimosis en tronco y extremidades, hirsutismo moderado y alopecia andrógina (Ludwig II); abdomen globuloso; se palpa masa en hipocondrio derecho. Analítica: PRL $40 \mathrm{ng} / \mathrm{mL}$, FSH/LH 0,37/0,32 mlU/mL, androsteneidona $>10 \mathrm{ng} / \mathrm{mL}$, DHEAS $695 \mathrm{ug} / \mathrm{dL}$, cortisol $8 \mathrm{am} / 11 \mathrm{pm}>50 \mathrm{ug} / \mathrm{dL}$, aldosterona $>500$, IFG-1: $62 \mathrm{ng} / \mathrm{mL}$, testosterona libre 2,08 pg/mL, CLU-1: $1141 \mathrm{ug} / \mathrm{d}, \mathrm{CLU}-2: 1178 \mathrm{ug} / \mathrm{d}, \mathrm{CLU}-3>1911 \mathrm{ug} / \mathrm{d}$, PFT normal. TEM de adrenales: masa heterogénea dependiente de la adrenal derecha de $12 \times 10 \mathrm{~cm}$, de contornos lobulados, contacta sin infiltrar el hígado; adrenal

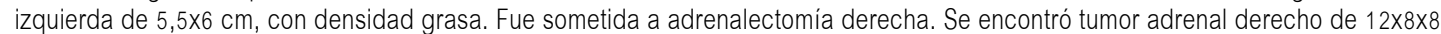
cm bien vascularizado. AP: carcinoma adrenal, grado histológico III de Furhman, con áreas focales de necrosis en 15\% del tumor,

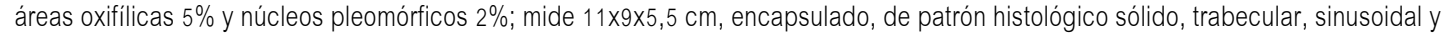
microacinar, que infiltra cápsula tumoral y se extiende focalmente a tejido graso periadrenal. Microembolia tumoral venosa y linfática. Remanente adrenal de aspecto hipotrófico. Ki-67 (+) en 10\%, S100 (-), CD34 (+). La paciente fue derivada al servicio de oncología para estadiaje y manejo.

Discusión y conclusiones: El CAC es una neoplasia poco frecuente, agresiva y con mal pronóstico. Representa $\leq 0,2 \%$ de todos los tumores malignos, con pronóstico pobre y supervivencia media de $<30 \mathrm{~m}$. Se estima una incidencia global de 0,5 a 2/1 millón de casos reportados anualmente en todo el mundo. Muestra una ligera preferencia en mujeres y una distribución por edades bimodal, con el primer pico en niños $<5$ años y el segundo pico en la $4^{\circ}$ a $5^{\circ}$ décadas de la vida. Deriva de las células de la corteza adrenal. En la tumorigénesis, puede conducir a exceso de producción hormonal en 60 a 70\% de los pacientes adultos, produciendo según la estirpe afectada Sd. de Cushing, Sd. de Conn, virilización o feminización. Series más recientes señalan la existencia de secreción hormonal hasta en $79 \%$; presentan pronóstico pobre y cuentan con pocas opciones de tratamiento según el estadiaje de la enfermedad. 


\title{
Mujer con amiloidosis primaria sistémica con bocio amiloide y carcinoma papilar de tiroides metastásico
}

\author{
David Liviac Cabrera', José Paz Ibarra', Marialejandra Delgado Rojas', \\ Natali Jáuregui Macedo', Vitalia Ranilla Seguín', Sofía Sáenz Bustamante", \\ José Somocurcio Peralta \\ ${ }^{1}$ Servicio de Endocrinologia y Metabolismo, Hospital Edgardo Rebagliati Martins. \\ ${ }^{2}$ Departamento de Anatomia Patológica, Hospital Edgardo Rebagliati Martins.
}

\begin{abstract}
Objetivo: Presentar un caso de carcinoma papilar de tiroides (CPT) metastásico en el contexto de bocio amiloide en una paciente con amiloidosis sistémica.

Metodología: Se presenta las características clínicas y paraclínicas de una paciente con amiloidosis sistémica y CPT metastásico.

Resultados: Mujer de 43 años ingresa por Emergencia por acentuación de cuadro clínico de 8 meses de evolución caracterizado por orina espumosa y anasarca, con diagnóstico de síndrome nefrótico por amiloidosis renal en otro nosocomio. Durante la hospitalización, se evidencia proteinuria 7,33 g/dia. Albúmina $1,36 \mathrm{~g} / \mathrm{dL}, \mathrm{Hb} 13,3 \mathrm{~g} / \mathrm{dL}, \mathrm{U} 32 \mathrm{mg} / \mathrm{dL}, \mathrm{Cr} 1,29 \mathrm{mg} / \mathrm{dL}$, depuración de $\mathrm{Cr} 33 \mathrm{~mL} / \mathrm{min}$, proteinuria de Bence Jones (+). AMO: Plasmocitos 12\%, no concluyente de mieloma múltiple. Inmunofijación en suero: Componente monoclonal IgG tipo lambda. Ecocardiograma: Patrón de llenado restrictivo, hipertrofia concéntrica moderada de VI, con zonas a nivel de septum sugerentes de infiltración. TEM cérvico-torácica: Efusión pleural bilateral moderada. Engrosamiento intersticial difuso con opacidades nodulares bilaterales pulmonares menores de $1 \mathrm{~cm}$; tiroides aumentada de volumen, con nódulo en LTI, parcialmente

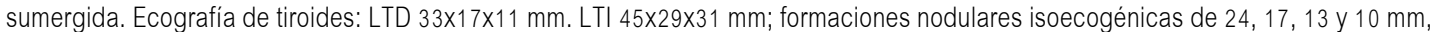
calcificaciones asociadas irregulares de 14 y $7 \mathrm{~mm}$, microcalcificaciones dispersas. Istmo $3 \mathrm{~mm}$. Ganglios de cadena cervical superior de morfología conservada, de 16x4 mm y 14x4 mm, bilaterales. BAAF: Positivo a malignidad. Evaluada por Hematologia: Amiloidosis primaria sistémica con compromiso renal y cardiaco. Inicia quimioterapia, con respuesta inicial favorable evidenciada por disminución de valores de proteinuria, y posteriormente cursa con evolución tórpida por compromiso renal y cardiaco. Fue sometida a tiroidectomía total más disección cervical, cuya evaluación patológica concluyó: CPT variedad folicular $100 \%$ no encapsulado que infiltra la cápsula tiroidea, sin evidencia de embolia linfática, que mide $10 \mathrm{~mm}$, ubicado en LTI. Tiroides remanente: bocio amiloide, IHQ: $\mathrm{Tg}(+)$, rojo congo(+). Referida al Servicio de Endocrinología para terapia ablativa. Perfil preablativo: TSH $>75 \mathrm{uUI} / \mathrm{mL} ; \mathrm{Tg} 83 \mathrm{ng} / \mathrm{mL} ; \mathrm{AbTg}<20 \mathrm{UI} /$ $\mathrm{mL}$. Recibe dosis corregida a función renal de 1131 de $70 \mathrm{mCi}$, cuyo rastreo corporal total posterior a terapia fue positivo en cuello y pulmones. Actualmente, en controles por consultorio externo de Endocrinología, Nefrología, Cardiología y Hematología.
\end{abstract}

Discusión y conclusiones: La amiloidosis constituye un grupo heterogéneo de enfermedades caracterizadas por el depósito extracelular de sustancia amiloide. Puede ser primaria, si no está asociada a otras enfermedades (excepto mieloma múltiple) o secundaria, si está asociada a procesos crónicos inflamatorios o infecciosos. Los órganos más frecuentemente afectados son el riñón, el corazón y el aparato digestivo. Si bien la afectación tiroidea es relativamente frecuente, presentándose en alrededor de 50 a $80 \%$ de los casos de amiloidosis, principalmente secundaria, la coexistencia de bocio amiloide y carcinoma diferenciado de tiroides es extremadamente rara, existiendo menos de 10 casos reportados en la literatura. La IHQ en el diagnóstico tiene un papel importante, pues la positividad en la tinción de rojo congo y Tg permite confirmar dicha asociación. 


\title{
Hipertrigliceridemia severa en un paciente de 36 años con cetoacidosis diabética al debut de diabetes mellitus tipo 2
}

\author{
Natali Jáuregui Macedo', Sofía Sáenz Bustamante", José Paz Ibarra', David Liviac \\ Cabrera', Marialejandra Delgado Rojas ${ }^{1}$, Vitalia Ranilla Seguín ${ }^{1}$ \\ ${ }^{1}$ Servicio de Endocrinologia y Metabolismo, Hospital Edgardo Rebagliati Martins, Lima, Perú.
}

\begin{abstract}
Objetivo: Presentar un caso de hipertrigliceridemia (HTG) severa en un paciente con CAD, al debut de DM2.
Metodología: Se presenta las características clínicas y paraclínicas de un paciente con HTG severa en un paciente con CAD, al debut de DM2.

Resultados: Varón de 36 años con diagnóstico de rosácea; padre, con DM2 desde los 40 años, llegó a pesar $110 \mathrm{~kg}$. IMC 35,5 kg/m2 hasta hace 9 meses; desde entonces refiere disminución de peso progresivo de $20 \mathrm{~kg}$. Hace 4 meses se agrega polidipsia, poliuria, polifagia y nicturia que va incrementándose. Dos dias antes del ingreso presenta hiporexia, náuseas, dolor abdominal y vómitos ( 8 a 9 episodios/d). Acudió a clínica particular, donde diagnostican CAD (pH 7,1, HCO3 3,5, Na+ $139 \mathrm{mEq} / \mathrm{L}, \mathrm{K}+4 \mathrm{mEq} / \mathrm{L}, \mathrm{G} 400 \mathrm{mg} \%$, cetonuria (+), glucosuria (+); amilasa, enzimas cardiacas, perfil hepático en límites normales). Recibió tratamiento consistente en hidratación e infusión de insulina EV, siendo trasferido luego de $12 \mathrm{~h}$ a la emergencia del HNERM. Al examen: IMC 30; no xantomas; lipemia retinalis en AO. Analítica: G 234 mg\%, pH 7,38, HbA1c 14,1\%, péptido C 1,27 ng/mL, ChT/HDL/Tg 922/16/2344 mg/dL; TSH $0,6 \mathrm{uUI} / \mathrm{mL} ; \mathrm{T} 4 \mathrm{~L} 1 \mathrm{ng} / \mathrm{dL}$; hemograma, pruebas de función hepática y renal, amilasa/lipasa sin alteraciones. Se indicó hidratación EV y VO, dieta vegetariana estricta por 5 dias y luego dieta por conteo de CHO; insulinoterapia SC mixta (NPH $40 \mathrm{UI} / \mathrm{d}+\mathrm{R} 15 \mathrm{UI} / \mathrm{d}$ ), metformina $2550 \mathrm{mg} / \mathrm{d}$, terapia combinada con atorvastatina $40 \mathrm{mg} / \mathrm{d}$ y genfibrozilo $1200 \mathrm{mg} / \mathrm{d}$. Al $5^{\circ}$ día de hospitalizado: ChT/ HDL/VLDL/Tg: 658/27/219/1 096 mg\%; el 10ªia: ChT/HDL/VLDL/Tg: 322/30/63/314 mg\% y al alta (15d): ChT/HDL/VLDL/Tg/LDL: 196/30/36/179/124 mg\%.

Discusión y conclusiones: La HTG es una anomalía frecuente del metabolismo lipídico. Las formas primarias suponen $<5 \%$ de las HTG. La mayoria de los pacientes con HTG tiene al menos un factor subyacente. Sin embargo, no todos los pacientes con una exposición similar a los factores desencadenantes desarrollan el mismo grado de dislipidemia. Aquellos pacientes con concentraciones de triglicéridos $>2000 \mathrm{mg} \%$ prácticamente tienen una HTG secundaria asociada a una de origen genético. Entre las causas secundarias, hay varias que podrian causar HTG grave $(>500 \mathrm{mg} \%)$, entre las cuales se destacan la DM y la CAD, alcohol, hipotiroidismo y medicamentos (estrógenos, inhibidores de proteasa, olanzapina, mirtazapina e isotretinoína). En los pacientes con Tg >1 $000 \mathrm{mg}$ existe un riesgo incrementado de desarrollar pancreatitis aguda, por lo que su manejo exige una disminución rápida y efectiva de Tg, habiéndose reportado el uso de insulina o heparina en infusión EV y plasmaféresis. En nuestro caso, el régimen nutricional estricto con el manejo de la glicemia y terapia hipolipemiante combinada permitió normalizar la glicemia y casi todos los parámetros lipoproteicos.
\end{abstract}




\title{
Control metabólico en cohorte de pacientes diabéticos atendidos en el Programa de Diabetes del Hospital Nacional Dos de Mayo, entre 2011 y 2013
}

\author{
Harold Torres Aparcana', Hugo Arbañil Huamán", Rosa Pando Álvarez', \\ Dante Gamarra Gonzales ${ }^{1}$, Rosa Agüero Zamora', Jaime Pajuelo Ramírez' \\ Hospital Nacional Dos de Mayo. Área de investigación: Diabetes.
}

\begin{abstract}
El Programa de Diabetes "Héctor Valdivia Carpio" del Hospital Nacional Dos de Mayo (HNDM) fue uno de los pioneros en brindar educación gratuita, evaluación para la prevención del pie diabético, asesoría nutricional y atención en podología, con la finalidad de obtener un buen control en los pacientes con diabetes, disminuyendo el riesgo de complicaciones y mejorando su calidad de vida.

Objetivo: Determinar el grado de control metabólico en la cohorte de pacientes atendidos en el Programa de Diabetes del HNDM entre enero 2011 y diciembre 2013

Metodología: Estudio transversal, analítico y observacional. Se invitó a los pacientes con diagnóstico de diabetes mellitus atendidos ambulatoriamente en el Servicio de Endocrinologia "Fausto Garmendia Lorena" a participar del Programa de Diabetes del HNDM. La cohorte estuvo conformada por aquellos pacientes que hubieran tenido por lo menos 2 controles en el programa, entre enero de 2011 y diciembre de 2013. Se excluyó a las gestantes y menores de 18 años. El grado de control fue determinado según las recomendaciones de la Asociación Americana de Diabetes (ADA), del 2014. Se evaluó el IMC, perímetro abdominal, presión arterial (PA), su último control de glucosa en ayunas (GA), HbA1c y perfil lipídico.
\end{abstract}

Para el análisis estadístico se empleó el programa SPSS versión 18. Se determinó la diferencia de medias mediante la prueba T de student; el coeficiente de Pearson fue usado para estimar la correlación entre la HbA1c y las otras variables; y se consideró estadísticamente significativo un $p<0,05$.

Resultados: Se evaluó 843 pacientes; 562 eran de sexo femenino (66,7\%). La edad promedio fue 60,9 $\pm 10,7$ años; la edad de las mujeres fue en promedio $60,6 \pm 10,7$ años y de los varones $61,7 \pm 10,9$ años, sin haber diferencia significativa $(p=0,185)$. El tiempo de diagnóstico de diabetes mellitus fue $8,2 \pm 8,0$ años. El tiempo de seguimiento promedio fue 10,9 $\pm 7,3$ meses, siendo equivalente a 767 personas-año. El número de controles en el programa fue en promedio $6,9 \pm 4,9$.

El IMC promedio fue $28,8 \pm 4,7$. De acuerdo a la clasificación de la OMS, $0,4 \%$ tenía peso bajo, $18,6 \%$ tenía peso normal, $45,4 \%$ sobrepeso y $35,6 \%$ obesidad. En las mujeres, el IMC fue en promedio $29,3 \pm 4,8$ y en los varones $27,8 \pm 4,2$, siendo esta diferencia estadisticamente significativa $(p<0,001)$. El perímetro abdominal promedio fue $98,1 \pm 10,7 \mathrm{~cm}$; en los varones fue $99,3 \pm 10,7 \mathrm{~cm}$ y en las mujeres $97,4 \pm 10,6 \mathrm{~cm}$, sin diferencia significativa $(p=0,14)$.

El promedio de glucosa en ayunas en el último control de los pacientes fue 130,6 $48,4 \mathrm{mg} / \mathrm{dL} ; 62,5 \%$ tenía entre 70 y $130 \mathrm{mg} / \mathrm{dL}$. El promedio de la última medición de $\mathrm{HbA} 1 \mathrm{C}$ fue $7,5 \pm 2,2 \%$ y $52,8 \%$ de los pacientes tenía valores de $\mathrm{HbA} 1 \mathrm{C}$ menores a $7 \%$. La diferencia entre el último y el primer control de HbA1c fue $-0,25 \pm 2,2 \%$. El $70,4 \%$ tenia PA $<140 / 80 \mathrm{mmHg}$. El $63,9 \%$ tenía colesterol total $<200 \mathrm{mg} / \mathrm{dL}, 26,3 \%$ colesterol $\mathrm{HDL}>50 \mathrm{mg} / \mathrm{dL}, 42,0 \%$ colesterol $\mathrm{LDL}<100 \mathrm{mg} / \mathrm{dL}$ y $51,9 \%$ triglicéridos $<150 \mathrm{mg} / \mathrm{dL}$. La HbA1c se correlacionó inversamente con la edad $(r=-0,10 ; p=0,048)$ y con el número de controles en el Programa de Diabetes $(r=-0,19$; $p<0,001)$, y directamente con el tiempo de diagnóstico de diabetes mellitus ( $r=0,32 ; p<0,001)$, con el colesterol total $(r=0,13 ; p=0,02)$, triglicéridos $(r=0,14 ; p=0,01)$, PA sistólica $(r=0,11 ; p=0,02)$ y PA diastólica $(r=0,11 ; p=0,002)$.

Conclusiones: Dos tercios de los pacientes atendidos en el Programa de Diabetes del HNDM fueron mujeres. Aproximadamente el $80 \%$ tenía sobrepeso u obesidad. El $62,5 \%$ y el $52,8 \%$ tenían GA y HbA1C dentro del rango recomendado por la ADA respectivamente. Cerca de $70 \%$ tenía PA<140/80 mmHg, mientras $42 \%$ tenía el colesterol LDL por debajo de $100 \mathrm{mg} / \mathrm{dL}$.

Financiamiento: Recursos propios. 


\title{
Osteoartropatía neuropática de Charcot en un adolescente con diabetes tipo I: reporte de caso
}

\author{
Pedro Aro ${ }^{1}$, Elena Tapia ${ }^{1}$, Helard Manrique ${ }^{1}$ \\ ${ }^{1}$ Médico. Centro de expertos en Diabetes Obesidad y Nutrición CIDON.
}

\begin{abstract}
La osteoartropatía de Charcot o pie diabético de Charcot es una de las complicaciones agudas-crónicas de la diabetes, que pone en riesgo una extremidad, afectando hueso, articulación y tejido blando del pie; puede ser confundido con diversas patologías infecciosas, traumáticas e incluso quirúrgicas, siendo su principal causa la neuropatía diabética.

Objetivo: Se reporta el tratamiento farmacológico y el curso clínico de un adolescente con diabetes tipo1 que cursó con osteoartropatía neuropática de Charcot.

Caso Clínico: Paciente varón de 17 años con diagnóstico de diabetes tipo 1 desde los 18 meses de edad. Tenía antecedente de hipotiroidismo y neuropatía diabética, con tratamiento de insulina Lispro $5 \mathrm{UI} / 7 \mathrm{UI} / 5 \mathrm{UI}$ e insulina glargina $20 \mathrm{UI} / 20 \mathrm{UI}$, gabapentina 5 $400 \mathrm{mg}$ día, amitriptilina $25 \mathrm{mg}$ al día y levotiroxina $100 \mathrm{ug} / \mathrm{dia}$. Acudió por presentar poliuria, polifagia, cuadro de dolor intenso en miembros inferiores, que lo llevaron a la postración; además, presentaba insomnio permanente y bajada de peso aproximadamente 5 kilos en el último mes. Al examen físico tenía la piel seca, dolor a la palpación, eritema con aumento de volumen en miembros inferiores, reflejos rotulianos disminuidos, pulsos pedios presentes, deformación de ambos pies y una escala de dolor en 10. En los exámenes de laboratorio: glucosa basal en $293 \mathrm{mg} / \mathrm{dL}, \mathrm{HbA} 1 \mathrm{C} 14,2 \%$, TSH 1,4, triglicéridos $72 \mathrm{mg} / \mathrm{dL}$, colesterol total $160 \mathrm{mg} / \mathrm{dL}$, creatinina $0,77 \mathrm{mg} / \mathrm{dL}$. El estudio neurofisiológico mostró evidencia de polineuropatía mixta sensitiva motora axonal de miembros inferiores. Además, en la evaluación oftalmológica presentó una retinopatía proliferativa. El manejo farmacológico instaurado fue tramadol $50 \mathrm{mg}$ cada 6 horas, pregabalina $300 \mathrm{mg}$ cada 12 horas, amitriptilina $25 \mathrm{mg}$ al día, con insulina glargina $32 \mathrm{UI}$ en la noche, insulina Lispro $8 \mathrm{UI} / 8 \mathrm{UI} / 8 \mathrm{UI}$, levotiroxina $100 \mathrm{ug} / \mathrm{dia}$. En el seguimiento a los 2 meses: HbA1C 12,5, glucosa basal $110 \mathrm{mg} / \mathrm{dL}$. El paciente refirió disminución del dolor, con una escala de dolor en 2.

Conclusiones: La osteoartropatía neuropática de Charcot es una complicación severa y compleja en pacientes con diabetes y neuropatía. Esta condición en poco frecuente y debe ser considerada en adolescentes con larga duración de la diabetes. La deformidad del pie en mecedora es una manifestación tardía y grave y puede ser evitada con un reconocimiento clínico temprano de esta entidad. La terapia intensiva del manejo de la diabetes con un buen control glicémico y evitar la carga del pie es el pilar de la prevención de esta complicación.
\end{abstract}

Financiamiento: Recursos propios. 


\title{
Niveles séricos de lípidos en adolescentes con obesidad
}

\author{
Pedro Aro ${ }^{2}$, Elena Tapia ${ }^{3}$, Helard Manrique ${ }^{1}$ \\ ${ }^{1}$ Médico. Hospital Arzobispo Loayza. Servicio de Endocrinología. \\ ${ }^{2}$ Médico. Hospital Cayetano Heredia. Centro de Hemoterapia y Banco de Sangre. \\ ${ }^{3}$ Médico. Centro de expertos en Diabetes Obesidad y Nutrición CIDON.
}

\begin{abstract}
La prevalencia de obesidad en adolescentes se ha incrementado notablemente en las últimas décadas, de tal manera que se ha convertido en un problema de salud pública. Este incremento de la prevalencia aumenta paralelamente los factores de riesgo cardiovascular, existiendo una fuerte evidencia entre la alteración del perfil lipídico y la enfermedad cardiovascular en niños y adolescentes.
\end{abstract}

Objetivo: Determinar los niveles séricos de lípidos en adolescentes obesos que acuden al servicio de endocrinología del Hospital Arzobispo Loayza durante el año 2013.

Metodología: Se realizó un estudio de corte transversal descriptivo. Se revisó 144 historias clínicas de adolescentes obesos, entre 10 y 17 años, de los cuales se tomó datos de edad, sexo, raza, medida de peso y talla, medida de circunferencia abdominal, presión arterial, HDL, LDL, triglicéridos y colesterol total. Los niveles de lípidos fueron valorados según las recomendaciones de The National Cholesterol Education Program (NCEP) Expert Panel on Cholesterol Levels in Children. Los datos son presentados en porcentajes (\%) para las variables categóricas y se llevaron a cabo en el programa STATA versión 10. El proyecto fue aprobado por el comité de ética de la Universidad Peruana Cayetano Heredia.

Resultados: Se incluyó a 144 adolescentes con diagnóstico de obesidad, de los cuales 73 (50,7\%) eran mujeres y $71(49,3 \%)$ eran hombres, con edad promedio de 13,1 años. Se obtuvo hipercolesterolemia en $26(18,1 \%)$ adolescentes, HDL disminuido en $29(20,1 \%)$ adolescentes, LDL elevado en 29 (20,1\%) e hipertrigliceridemia en $72(50 \%)$ adolescentes.

Conclusiones: El estudio muestra una alta prevalencia de los niveles séricos del perfil lipídico en adolescentes con obesidad, por lo que es necesario que el personal de salud detecte oportunamente el problema y oriente a la familia para evitar factores de riesgo para enfermedad cardiovascular desde la infancia.

\begin{tabular}{cccc} 
& Deseable & Riesgo potencial & Riesgo alto \\
Colesterol total & $69(47,9 \%)$ & $49(34,0 \%)$ & $26(18,1 \%)$ \\
HDL & $87(60,4 \%)$ & $28(19,5 \%)$ & $29(20,1 \%)$ \\
LDL & $43(29,9 \%)$ & $72(50,0 \%)$ & $29(20,1 \%)$ \\
Triglicéridos & $31(21,5 \%)$ & $41(28,5 \%)$ & $72(50,0 \%)$ \\
\hline
\end{tabular}

Financiamiento: Recursos propios. 


\title{
Prevalencia de obesidad en donantes de sangre en un hospital general
}

\author{
Pedro Aro², Elena Tapia³ ${ }^{3}$ Helard Manrique ${ }^{1}$, Julio Vidal² \\ ${ }^{1}$ Médico. Hospital Arzobispo Loayza, Servicio de Endocrinología. \\ ${ }^{2}$ Médico. Hospital Cayetano Heredia. Centro de Hemoterapia y Banco de Sangre. \\ ${ }^{3}$ Médico. Centro de expertos en Diabetes Obesidad y Nutrición CIDON.
}

La obesidad constituye un problema de salud pública que ha sido calificada como la 'epidemia del siglo', y a la que se ha destinado una gran cantidad de recursos económicos y humanos para su manejo, control y prevención.

Objetivo: Determinar la prevalencia de obesidad en donantes de sangre que acuden al hospital Cayetano Heredia.

Metodología: Estudio de tipo transversal, que incluyó 510 donantes de sangre calificados como aptos, entre 18 y 55 años, que acudieron al Centro de Hemoterapia y Banco de Sangre del hospital Cayetano Heredia durante los meses de mayo y junio de 2014. Se definió obesidad a un IMC $\geq 30$, sobrepeso IMC $\geq 25$ a $<30$ y peso normal $<25$. Los datos son presentados en porcentajes (\%) para las variables categóricas y fueron llevados al programa STATA versión 10.

Resultados: Se incluyó a 510 donantes, de los cuales 187 (36,7\%) eran mujeres y 323 (63,3\%) hombres. Se obtuvo una prevalencia de sobrepeso de 44,3\% y de obesidad de 30,9\%; eran mujeres con sobrepeso 82 y hombres 144; eran mujeres con obesidad 61 y hombres 97 .

\begin{tabular}{ccccc} 
& \multicolumn{3}{c}{ Índice de masa corporal } & \\
\cline { 2 - 4 } Sexo & Normal & Sobrepeso & Obesidad & Total \\
Femenino & 44 & 82 & 61 & 187 \\
& 23,5 & 43,9 & 32,6 & 100,0 \\
\hline Masculino & 82 & 144 & 97 & 323 \\
& 25,4 & 44,6 & 30,0 & 100,0 \\
\hline Total & 126 & 226 & 158 & 510 \\
& 24,7 & 44,3 & 30,9 & 100.0 \\
\hline
\end{tabular}

Conclusiones: Se halló una alta prevalencia de obesidad en donantes de sangre, incluso mayor a la prevalencia de nuestro país. Estos datos en conjunto apoyan el concepto de que el banco de sangre puede ser un lugar útil para el seguimiento de la población que presenta obesidad y posiblemente la introducción de intervenciones hacia el mantenimiento de un índice de masa corporal saludable.

Financiamiento: Recursos propios. 